\title{
Neurophotonics
}

\section{Neurophotonics applications to motor cortex research: a review}

Benjamin A. Suter

Naoki Yamawaki

Katharine Borges

Xiaojian Li

Taro Kiritani

Bryan M. Hooks

Gordon M. G. Shepherd

\section{SPIE.}




\title{
Neurophotonics applications to motor cortex research: a review
}

\author{
Benjamin A. Suter, ${ }^{a}$ Naoki Yamawaki, ${ }^{a}$ Katharine Borges, ${ }^{a}$ Xiaojian Li, ${ }^{\text {a }}$ Taro Kiritani, ${ }^{a}$ \\ Bryan M. Hooks, ${ }^{b}$ and Gordon M. G. Shepherd ${ }^{\mathrm{a}, *}$ \\ ${ }^{a}$ Northwestern University, Feinberg School of Medicine, Department of Physiology, Chicago, Illinois 60611 \\ ${ }^{\mathrm{b}}$ Howard Hughes Medical Institute, Janelia Farm Research Campus, Ashburn, Virginia 20147
}

\begin{abstract}
Neurophotonics methods offer powerful ways to access neuronal signals and circuits. We highlight recent advances and current themes in this area, emphasizing tools for mapping, monitoring, and manipulating excitatory projection neurons and their synaptic circuits in mouse motor cortex. () The Authors. Published by SPIE under a Creative Commons Attribution 3.0 Unported License. Distribution or reproduction of this work in whole or in part requires full attribution of the original publication, including its DOI. [DOI: 10.1117/1.NPh.1.1.011008]
\end{abstract}

Keywords: optogenetics; motor cortex; pyramidal neuron; corticospinal; mapping.

Paper 14009VSSR received Feb. 24, 2014; revised manuscript received Apr. 16, 2014; accepted for publication Apr. 18, 2014; published online Jun. 13, 2014.

\section{Introduction}

For neuroscientists studying the connectivity and function of neuronal circuits, the growing collection of photonics and optical tools offers exciting new possibilities to assess how signals are integrated in cells, how cells are interconnected to form circuits, and how neuronal activity relates to behavior. In this review, we highlight recent advances in the use of light-based methods for investigating cortical circuits in motor cortex.

Two basic approaches are often taken to try to decipher how motor cortex relates to movements: (1) stimulate or silence motor cortex and measure the resulting effect on skeletal muscle activity and body movements and (2) record motor cortex activity during motor behavior and assess how this correlates with movement parameters. With new and emerging neurophotonics and optogenetic methods, these two basic types of approaches are being pursued at even higher levels of specificity and spatiotemporal precision, and can even be combined in the same experiment.

In this review, we first consider how projection neurons in motor cortex, including key cell types such as corticospinal neurons, can be accessed experimentally. We then describe new ways to monitor and manipulate the activity of motor cortex neurons in vivo. We conclude with a consideration of slicebased tools for mapping synaptic connections among motor cortex neurons.

\section{New Ways to Access Motor Cortex Projection Neurons}

There is great interest in understanding how the different classes of projection neurons, such as corticospinal neurons, are involved in motor control. ${ }^{1-4}$ Projection neurons fall into distinct classes based on their long-range axonal arborization patterns (reviewed in Ref. 5). By injecting retrograde labeling reagents into the projection territories, the different classes of pyramidal neurons in motor cortex can be selectively labeled. Fluorescent

*Address all correspondence to: Gordon M. G. Shepherd, E-mail: g-shepherd@ northwestern.edu microspheres ${ }^{6}$ and other biologically inert retrograde tracers have been extensively used to label cortical projection neurons for anatomical and physiological analyses. ${ }^{7,8}$ Viruses with native or recombinantly engineered tropism for axons can be used to selectively transfect projection neurons with genes of interest. Recombinant rabies virus methods have been developed for a range of such applications. ${ }^{9,10}$ Retrograde transfection with deletion-mutant rabies viruses has been used to label corticospinal and corticostriatal neurons with channelrhodopsin-2 (ChR2) for circuit-mapping of intracortical connectivity in motor cortex slices. ${ }^{11}$ Rabies viruses can also be used to deliver genetically encoded calcium indicators (GECIs) and other constructs enabling projection-specific monitoring and manipulation of neuronal activity. ${ }^{12-14}$

An important development in recent years has been the development of Cre driver lines-transgenic mouse lines with cell-type-specific expression of cre recombinase restricted to particular subsets of cortical neurons-including lines specific for major classes of GABAergic interneurons ${ }^{15}$ and excitatory projection neurons. ${ }^{16}$ These lines greatly extend the experimental possibilities for investigating motor cortical circuits and cortical mechanisms of motor control.

\section{In Vivo Monitoring of Neuronal Activity in the Motor Cortex of Head-Fixed Rodents}

The experimental paradigm of studying motor behavior in headfixed animals while recording and/or stimulating in motor cortex has long been a cornerstone of research on cortical mechanisms of motor control in monkeys. There has been much progress toward developing and refining comparable methods for rodents, ${ }^{17-20}$ including the design of apparatus enabling precise quantification of a variety of locomotor, vibrissal (whisking), forelimb, and other motor behaviors. ${ }^{21-24}$ The stability provided by head-fixation enables these approaches to be combined with optical (e.g., two-photon microscopy) and electrical (e.g., whole-cell recording) methods for measuring neuronal activity in motor cortex. Voltage-sensitive dye imaging can be performed on a wide scale across the exposed neocortex, revealing 
the spatiotemporal patterns of motor cortex responses to somatosensory stimuli. ${ }^{25}$ Synthetic calcium-sensitive dyes can be injected directly into the cortex in vivo, enabling activity to be monitored across large numbers of neurons with two-photon microscopy. ${ }^{26}$ GECIs are available with excellent sensitivity for reporting action potentials and multiple kinetic and wavelength options. ${ }^{27,28}$ With the viral methods and Cre lines mentioned above, there are now many possibilities for targeting GECI expression to cortical cell types of interest. Thus, calcium signals can be recorded from cell classes of interest in the motor cortex of awake and behaving mice moving (e.g., on a treadmill) during head-fixation. We expect this to be a powerful general paradigm that can be applied in many permutations to assess how different classes of motor cortex neurons are engaged during different types of motor behavior.

So far, layer $2 / 3$ pyramidal neurons have been the most extensively studied cell class in vivo in the motor cortex, being the most superficial excitatory neurons and, therefore, the easiest to image in vivo with two-photon microscopy. These studies are revealing the fine-scale organization of activity across groups of these neurons imaged during different types of motor behavior ${ }^{21,29-31}$ [Fig. 1(a)]. An ongoing challenge is to image deeper into the cortex, to sample activity in vivo in the somatic and basilar dendritic compartments of deeper-layer pyramidal neurons. ${ }^{32}$ Another challenge is to detect activity at the level of single axonal branches, and with axonal calcium imaging, it is possible to detect signals in motor cortex neurons' axons traveling in layer 1 of somatosensory cortex ${ }^{33}$ [Fig. 1(b)].

\section{In Vivo Manipulation of Neuronal Activity in the Motor Cortex of Head-Fixed Rodents}

The paradigms described above are also readily combinable with optogenetic methods for selectively increasing or decreasing activity in targeted cell classes. Activation by photostimulation of ChR2-expressing cells in transgenic mice has been used for topographic mapping of motor activity across the motor cortex ${ }^{37}$ and can even be performed transcranially. ${ }^{38}$ This line of investigation is yielding new insights into the cortical topography of the forelimb representation ${ }^{3,34,39}$ [Fig. 1(c)]. A similar approach has also been used in combination with voltage-sensitive dye imaging of the entire dorsal surface of the neocortex, providing a novel large-scale map of corticocortical connectivity in the intact brain. ${ }^{40,41}$

Optogenetic manipulation is compatible with head-fixed behavioral paradigms, mentioned in the previous section, enabling exploration of the functional roles of different motor cortex neurons. For example, activation and inactivation of corticospinal versus corticostriatal circuits may have distinct effects on an animal's ability to learn or perform motor tasks. In general, whereas activation methods usually rely on photostimulation of excitatory neurons transfected with ChR2, inactivation can be effected either by directly silencing excitatory neurons through photoactivation of a hyperpolarizing opsin, such as halorhodopsin, or by activating GABAergic neurons. For example, in a recent study, cortical activity was silenced transiently and focally, in mice with ChR2-expressing cortical interneurons, at an array of discrete cortical locations during a behavioral task; this innovative imaging-based approach to behavioral mapping revealed the cortical areas involved in different aspects of a motor task ${ }^{36}$ [Fig. 1(e)].

Optogenetic activation of ChR2-expressing neurons can be useful not only for assessing the effects on motor behavior but also as a tool for identifying (tagging) the neurons for subsequent analysis of monitored activity. ${ }^{42}$ This neurophotonics technique is conceptually similar to the electrophysiological method of antidromic activation to identify different classes of projection neurons in motor cortex, but with the added dimension of being applicable to any class of neuron that can be targeted to express ChR2.

\section{Brain Slice Circuit-Mapping of Motor Cortex Circuits}

Compared to experiments in intact animals, the acute brain slice preparation permits greater ease of access to neurons across all cortical layers, both in terms of targeting specific cells for pipette-based recording of membrane potential and for photostimulation and optical imaging.

Laser scanning photostimulation based on glutamate uncaging (glu-LSPS) has been used to topographically map local inputs to different classes of excitatory and inhibitory neurons in motor cortex and other frontal agranular areas. ${ }^{43-48}$ Glu-LSPS entails calibration and control experiments to quantify the efficacy of photoexcitability, allowing data to be pooled or compared across animals. . $^{47,49-51}$

ChR2-based photostimulation has also been used to assess cell-specific connectivity in motor cortex circuits, not only for local circuits but-because severed ChR2-expressing axons remain photoexcitable in brain slices ${ }^{52}$-also for longrange connections. ChR2 photostimulation can be combined with viral and genetic techniques to achieve cellular specificity. In addition to its utility for mapping static connectivity, ChR2 photostimulation can be performed at faster rates to generate dynamic barrages of synaptic inputs, an approach that has been applied to examine dendritic integration of intracortical inputs to corticospinal neurons. ${ }^{53}$

Subcellular ChR2-assisted circuit mapping (sCRACM) ${ }^{54}$ takes mapping to the level of dendrites. A cocktail of ion channel blockers in the bath solution prevents active propagation of spikes, but enables local depolarization of ChR2-expressing axons by the focused laser beam. Performed sequentially across an array of sites, this mode of direct photostimulation of presynaptic terminals generates a synaptic input map representing the dendritic locations of inputs from a specific presynaptic source [Fig. 1(d)].

Variability in ChR2 transfection efficiency, expression levels, and other factors often make it difficult or simply impossible to make quantitative comparisons across data from different animals. A common way around this problem is to record from multiple neurons per slice and to use one of the neurons (typically the strongest-responding one) as a fiduciary to which other neurons' responses can be compared. ${ }^{52,55}$ This normalization-based strategy means that connectivity is expressed in relative rather than absolute terms, but is nevertheless powerful for identifying systematic differences in circuit organization.

ChR2-based methods have been used to assess the relative targeting of inputs to motor cortex neurons from upstream cortical and thalamic sources. ${ }^{35,39,55}$ Similar approaches are also being applied to study the output pathways of motor cortex, such as its projections to auditory cortex ${ }^{56}$ and identified classes of medium spiny neurons in striatum. ${ }^{57}$ The general approach of long-range connectivity mapping at the synaptic level holds great promise for its applicability to the general goal of characterizing the cellular organization of synaptic circuits distributed on meso- and macroscopic scales across the neocortex. ${ }^{58}$ 
In vivo calcium-sensitive dye imaging (Dombeck et al., 2009)

(a1)

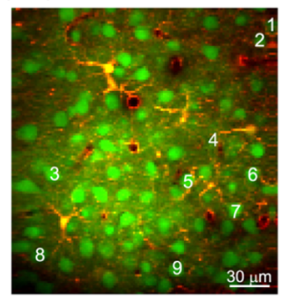

(a2)

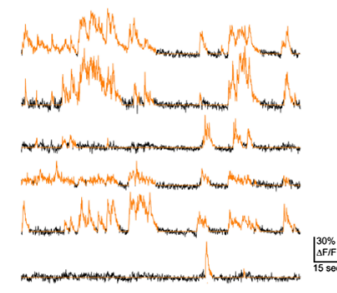

In vivo motor mapping (Harrison et al., 2012)

(c1) fixed * ketamine/xylazine

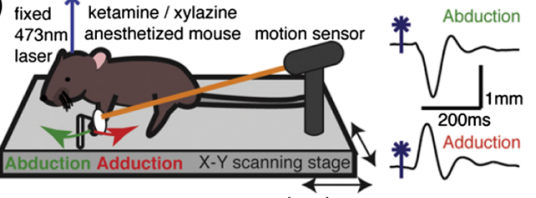

(c2)

(c3)

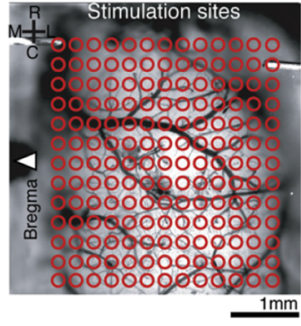

In vivo axonal calcium imaging (Petreanu et al., 2012)

(b1) Multiphoton
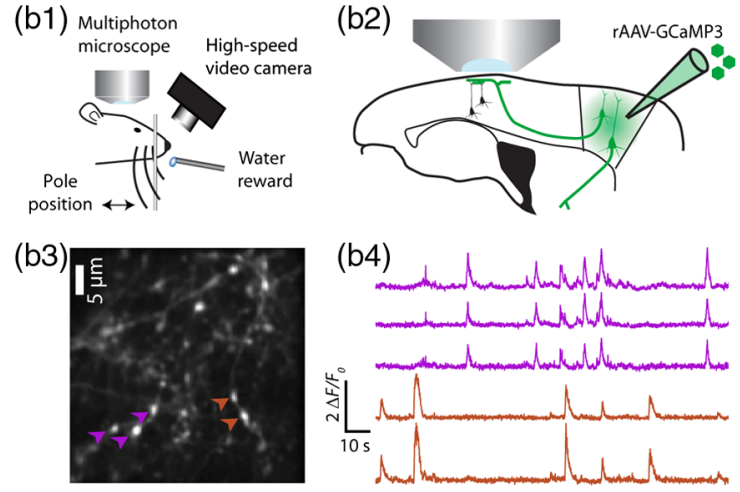

Ex vivo connectivity mapping with sCRACM (Mao et al., 2011) (d1)

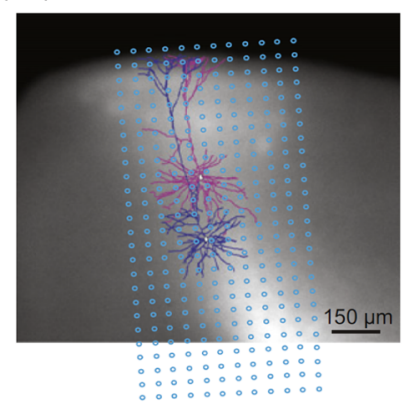

$(\mathrm{d} 2)$

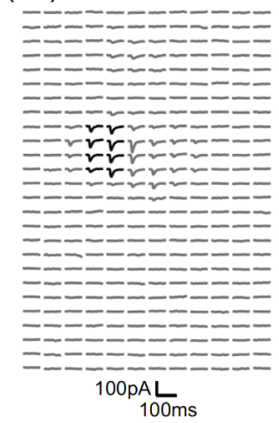

(e1)

In vivo behavioral mapping with focal cortical silencing (Guo et al., 2014)

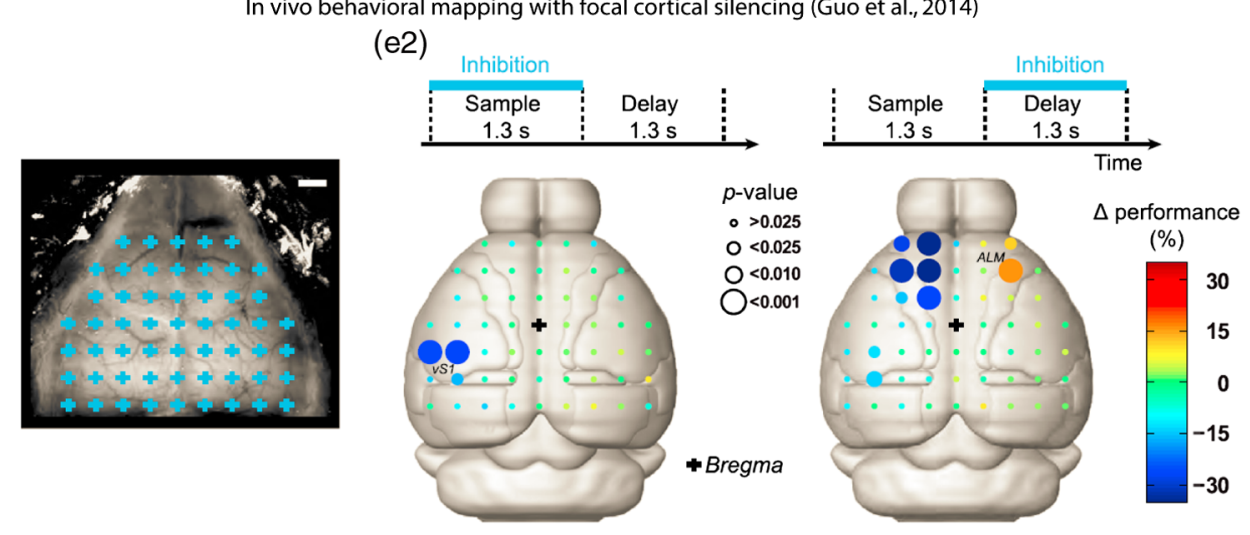

Fig. 1 Neurophotonics techniques for investigating neuronal activity and connectivity in motor cortex, and cortical involvement in sensorimotor processing. (a) In vivo calcium-sensitive dye imaging of activity in layer $2 / 3$ pyramidal neurons in mouse motor cortex. A synthetic calcium-sensitive dye was bulk-loaded into motor cortex, resulting in labeling of layer $2 / 3$ neurons (a1), and two-photon microscopy was used to image calcium transients, representing action potentials, across many neurons in the same field of view (a2). Reproduced with permission from Ref. 21. (b) In vivo axonal calcium imaging. Mice were trained to perform a whisking behavioral task during head fixation (b1). Axons of vibrissal motor cortex (vM1) neurons were labeled with a genetically encoded calcium indicator (rAAV-GCaMP3), and their axons projecting to vibrissal somatosensory cortex (vS1) were imaged with two-photon microscopy [(b2) and (b3)] to detect calcium transients as the mouse performed the motor task (b4). Images from K. Svoboda, modified from Ref. 33. (c) In vivo motor mapping. Mice expressing ChR2 in layer 5B neurons in motor cortex were placed on an apparatus for laser-stimulating the motor cortex while monitoring forelimb movements with a motion sensor (c1). By sequentially stimulating across a grid over the motor cortex (c2), a map of the forelimb movements was obtained (c3). Reproduced with permission from Ref. 34. (d) Ex vivo connectivity mapping. Recordings were made from vM1 neurons in layer 5A (magenta) and 5B (blue) and a photostimulus grid was oriented to span their dendritic arbors (d1). By photostimulating across the array of sites to excite ChR2-expressing presynaptic terminals of axons originating from $\mathrm{S} 1$, a subcellular-resolution map was obtained revealing the dendritic sites of long-range input from S1 to these L5A neurons in M1 (d2). Reproduced with permission from Ref. 35. (e) In vivo behavioral mapping. In mice in which cortical interneurons express ChR2, cortical sites were focally inhibited during a sensorimotor task (e1). Scale bar, $1 \mathrm{~mm}$. This approach identified roles for somatosensory cortex (e2, left) and anterior lateral motor cortex (e2, right) during different phases of the behavioral task. Reproduced with permission from Ref. 36. 
Optogenetic circuit-mapping methods thus dovetail nicely with ongoing large-scale efforts to map anatomical projections in the mouse. ${ }^{59,60}$

\section{Prospects}

The primate has long been the major model system for human motor cortex, and for good reasons: primates have highly evolved manual dexterity resembling that of humans, and can learn complex motor tasks. However, rodents, and particularly mice, offer many advantages. They enable ready access to monitoring and manipulating specific cell types of interest, and there have been substantial advances in developing motor behavioral paradigms, including head-fixed preparations. Optogenetic methods for primate cortex are rapidly evolving, ${ }^{61,62}$ and although challenges are substantial, they are not insuperable. Several groups are pursuing application of optogenetic activation and inactivation methods to investigate cortical control of eye movements. ${ }^{63,64}$ Virus-based strategies have been developed for retrograde transfection of cortical projection neurons in marmoset cortex. ${ }^{65}$ Recent efforts using a combination of synthetic calcium indicators, fiber-optic photometry, and intracortical microstimulation demonstrate how neurophotonics tools can provide new insight into longstanding questions about motor cortex organization in the nonhuman primate. ${ }^{66}$ Fiber-optic photonics methods are also proving powerful as a way to access signals in different classes of striatal neurons. ${ }^{67}$

Despite all the progress, there are still tools we lack. Although several methods-particularly glu-LSPS, sCRACM, monosynaptic viral tracing-are available for imaging convergent input connectivity in cortical circuits, methods for imaging the divergent output connections from a single neuron or a defined class of neurons have been much more limited. ${ }^{68}$ Recent innovations with anterograde trans-synaptic viral tracing methods are a promising development in this area. ${ }^{69,70}$ Methods are also limited for manipulating one and only one connection; that is, for manipulating the $\mathrm{A} \rightarrow \mathrm{B}$ connection alone, without affecting all the other outputs of $\mathrm{A}$ and/or all the other inputs to B. But there is rapid progress on these and many fronts. For example, though still limited, options for red-shifted and other spectral variants ${ }^{71-73}$ and for subcellular targeting ${ }^{74}$ of opsins and reporters continue to improve. Chemogenetic methods provide complementary tools to optogenetic approaches, extending the possibilities for manipulating specific cell classes located deep in the brain. ${ }^{75,76}$

Motor cortex is a major hub in the brain, both in terms of its circuit organization, with massive convergence of pathways into and divergence of projections out of the local circuits, and in terms of its functional roles, operating as a key node in the sensorimotor and cognitive systems regulating motivated behaviors. Neurophotonics approaches hold great promise for monitoring and manipulating the activities of key cell classes in motor cortex, offering new opportunities to tackle longstanding questions about the cellular mechanisms in motor cortex involved in controlling movements and volitional behavior. ${ }^{1,2}$

\section{Acknowledgments}

Funding support: National Institute of Neurological Disorders and Stroke of the National Institutes of Health (R01NS061963, R21NS087479) and the Whitehall Foundation Inc.

\section{References}

1. E. V. Evarts, Y. Shinoda, and S. P. Wise, Neurophysiological Approaches to Higher Brain Functions, John Wiley \& Sons, New York (1984).

2. C. G. Phillips and R. Porter, Corticospinal Neurones: Their Role in Movement, Academic Press, London (1977).

3. T.C. Harrison and T. H. Murphy, "Towards a circuit mechanism for movement tuning in motor cortex," Front. Neural Circuits 6, 127 (2012).

4. B. Pasquereau and R. S. Turner, "Primary motor cortex of the Parkinsonian monkey: differential effects on the spontaneous activity of pyramidal tract-type neurons," Cereb. Cortex 21(6), 1362-1378 (2011).

5. G. M. G. Shepherd, "Corticostriatal connectivity and its role in disease," Nat. Rev. Neurosci. 14(4), 278-291 (2013).

6. L. C. Katz, A. Burkhalter, and W. J. Dreyer, "Fluorescent latex microspheres as a retrograde neuronal marker for in vivo and in vitro studies of visual cortex," Nature 310(5977), 498-500 (1984).

7. A. Akintunde and D. F. Buxton, "Origins and collateralization of corticospinal, corticopontine, corticorubral and corticostriatal tracts: a multiple retrograde fluorescent tracing study," Brain Res. 586(2), 208-218 (1992).

8. A. M. Hattox and S. B. Nelson, "Layer V neurons in mouse cortex projecting to different targets have distinct physiological properties," J. Neurophysiol. 98(6), 3330-3340 (2007).

9. I. R. Wickersham et al., "Retrograde neuronal tracing with a deletionmutant rabies virus," Nat. Methods 4(1), 47-79 (2007).

10. I. R. Wickersham, H. A. Sullivan, and H. S. Seung, "Production of glycoprotein-deleted rabies viruses for monosynaptic tracing and highlevel gene expression in neurons," Nat. Protoc. 5(3), 595-606 (2010).

11. T. Kiritani et al., "Hierarchical connectivity and connection-specific dynamics in the corticospinal-corticostriatal microcircuit in mouse motor cortex," J. Neurosci. 32(14), 4992-5001 (2012).

12. F. Osakada et al., "New rabies virus variants for monitoring and manipulating activity and gene expression in defined neural circuits," Neuron 71(4), 617-631 (2011).

13. F. Osakada and E. M. Callaway, "Design and generation of recombinant rabies virus vectors," Nat. Protoc. 8(8), 1583-1601 (2013).

14. M. Ginger et al., "Revealing the secrets of neuronal circuits with recombinant rabies virus technology," Front. Neural Circuits 7, 2 (2013).

15. H. Taniguchi et al., "A resource of Cre driver lines for genetic targeting of GABAergic neurons in cerebral cortex," Neuron 71(6), 995-1013 (2011).

16. C. R. Gerfen, R. Paletzki, and N. Heintz, "GENSAT BAC cre-recombinase driver lines to study the functional organization of cerebral cortical and basal ganglia circuits," Neuron 80(6), 1368-1383 (2013).

17. C. Schwarz et al., "The head-fixed behaving rat-procedures and pitfalls," Somatosens. Mot. Res. 27(4), 131-148 (2010).

18. D. H. O'Connor et al., "Vibrissa-based object localization in head-fixed mice," J. Neurosci. 30(5), 1947-1967 (2010).

19. Z. V. Guo et al., "Procedures for behavioral experiments in head-fixed mice," PLoS One 9(2), e88678 (2014).

20. D. N. Hill et al., "Biomechanics of the vibrissa motor plant in rat: rhythmic whisking consists of triphasic neuromuscular activity," J. Neurosci. 28(13), 3438-3455 (2008).

21. D. A. Dombeck, M. S. Graziano, and D. W. Tank, "Functional clustering of neurons in motor cortex determined by cellular resolution imaging in awake behaving mice," J. Neurosci. 29(44), 13751-13760 (2009).

22. D. A. Dombeck et al., "Imaging large-scale neural activity with cellular resolution in awake, mobile mice," Neuron 56(1), 43-57 (2007).

23. Y. Isomura et al., "Microcircuitry coordination of cortical motor information in self-initiation of voluntary movements," Nat. Neurosci. 12(12), 1586-1593 (2009).

24. D. H. O'Connor et al., "Neural coding during active somatosensation revealed using illusory touch," Nat. Neurosci. 16(7), 958-965 (2013).

25. I. Ferezou et al., "Spatiotemporal dynamics of cortical sensorimotor integration in behaving mice," Neuron 56(5), 907-923 (2007).

26. C. Stosiek et al., "In vivo two-photon calcium imaging of neuronal networks," Proc. Natl. Acad. Sci. U. S. A. 100(12), 7319-7324 (2003). 
27. T. W. Chen et al., "Ultrasensitive fluorescent proteins for imaging neuronal activity," Nature 499(7458), 295-300 (2013).

28. L. L. Looger and O. Griesbeck, "Genetically encoded neural activity indicators," Curr. Opin. Neurobiol. 22(1), 18-23 (2012).

29. T. Komiyama et al., "Learning-related fine-scale specificity imaged in motor cortex circuits of behaving mice," Nature 464(7292), 1182-1186 (2010).

30. D. Huber et al., "Multiple dynamic representations in the motor cortex during sensorimotor learning," Nature 484(7395), 473-478 (2012).

31. R. Hira et al., "Spatiotemporal dynamics of functional clusters of neurons in the mouse motor cortex during a voluntary movement," J. Neurosci. 33(4), 1377-1390 (2013).

32. D. N. Hill et al., "Multibranch activity in basal and tuft dendrites during firing of layer 5 cortical neurons in vivo," Proc. Natl. Acad. Sci. U. S. A. 110(33), 13618-13623 (2013).

33. L. Petreanu et al., "Activity in motor-sensory projections reveals distributed coding in somatosensation," Nature 489(7415), 299-303 (2012).

34. T. C. Harrison, O. G. Ayling, and T. H. Murphy, "Distinct cortical circuit mechanisms for complex forelimb movement and motor map topography," Neuron 74(2), 397-409 (2012).

35. T. Mao et al., "Long-range neuronal circuits underlying the interaction between sensory and motor cortex," Neuron 72(1), 111-123 (2011).

36. Z. V. Guo et al., "Flow of cortical activity underlying a tactile decision in mice," Neuron 81(1), 179-194 (2014).

37. O. G. Ayling et al., "Automated light-based mapping of motor cortex by photoactivation of channelrhodopsin-2 transgenic mice," Nat. Methods 6(3), 219-224 (2009).

38. R. Hira et al., "Transcranial optogenetic stimulation for functional mapping of the motor cortex," J. Neurosci. Methods 179(2), 258-263 (2009).

39. R. Hira et al., "In vivo optogenetic tracing of functional corticocortical connections between motor forelimb areas," Front. Neural Circuits 7, 55 (2013).

40. M. H. Mohajerani et al., "Spontaneous cortical activity alternates between motifs defined by regional axonal projections," Nat. Neurosci. 16(10), 1426-1435 (2013).

41. D. H. Lim et al., "In vivo large-scale cortical mapping using channelrhodopsin-2 stimulation in transgenic mice reveals asymmetric and reciprocal relationships between cortical areas," Front. Neural Circuits 6, 11 (2012).

42. S. Q. Lima et al., "PINP: a new method of tagging neuronal populations for identification during in vivo electrophysiological recording," PLoS One 4(7), e6099 (2009).

43. N. Weiler et al., "Top-down laminar organization of the excitatory network in motor cortex," Nat. Neurosci. 11(3), 360-366 (2008).

44. J. Yu et al., "Local-circuit phenotypes of layer 5 neurons in motorfrontal cortex of YFP-H mice," Front. Neural Circuits 2, 6 (2008).

45. C. T. Anderson et al., "Sublayer-specific microcircuits of corticospinal and corticostriatal neurons in motor cortex," Nat. Neurosci 13(6), 739744 (2010).

46. A. Apicella et al., "Laminarly orthogonal excitation of fast spiking and low threshold spiking interneurons in mouse motor cortex," J. Neurosci. 32, 7021-7033 (2012).

47. B. M. Hooks et al., "Laminar analysis of excitatory local circuits in vibrissal motor and sensory cortical areas," PLoS Biol. 9(1), e1000572 (2011).

48. S. Qiu et al., "Circuit-specific intracortical hyperconnectivity in mice with deletion of the autism-associated met receptor tyrosine kinase," J. Neurosci. 31(15), 5855-5864 (2011).

49. D. Schubert et al., "Layer-specific intracolumnar and transcolumnar functional connectivity of layer V pyramidal cells in rat barrel cortex," J. Neurosci. 21(10), 3580-3592 (2001).

50. G. M. G. Shepherd, T. A. Pologruto, and K. Svoboda, "Circuit analysis of experience-dependent plasticity in the developing rat barrel cortex," Neuron 38(2), 277-289 (2003).
51. L. Wood and G. M. G. Shepherd, "Synaptic circuit abnormalities of motor-frontal layer $2 / 3$ pyramidal neurons in a mutant mouse model of Rett syndrome," Neurobiol. Dis. 38(2), 281-287 (2010).

52. L. Petreanu et al., "Channelrhodopsin-2-assisted circuit mapping of long-range callosal projections," Nat. Neurosci. 10(5), 663-668 (2007).

53. P. L. Sheets et al., "Corticospinal-specific HCN expression in mouse motor cortex: Ih-dependent synaptic integration as a candidate microcircuit mechanism involved in motor control," J. Neurophysiol. 106(5), 2216-2231 (2011).

54. L. Petreanu et al., "The subcellular organization of neocortical excitatory connections," Nature 457(7233), 1142-1145 (2009).

55. B. M. Hooks et al., "Laminar organization of long-range excitatory input to mouse motor cortex," J. Neurosci. 33(2), 748-760 (2013).

56. A. Nelson et al., "A circuit for motor cortical modulation of auditory cortical activity," J. Neurosci. 33(36), 14342-14353 (2013).

57. G. J. Kress et al., "Convergent cortical innervation of striatal projection neurons," Nat. Neurosci. 16(6), 665-667 (2013).

58. J. W. Bohland et al., "A proposal for a coordinated effort for the determination of brainwide neuroanatomical connectivity in model organisms at a mesoscopic scale," PLoS Comput. Biol. 5(3), e1000334 (2009).

59. B. Zingg et al., "Neural networks of the mouse neocortex," Cell 156(5), 1096-1111 (2014)

60. S. W. Oh et al., "A mesoscale connectome of the mouse brain," Nature 508(7495), 207-214 (2014).

61. O. Ruiz et al., "Optogenetics through windows on the brain in the nonhuman primate," J. Neurophysiol. 110(6), 1455-1467 (2013).

62. I. Diester et al., "An optogenetic toolbox designed for primates," Nat. Neurosci. 14(3), 387-397 (2011).

63. J. Dai, D. I. Brooks, and D. L. Sheinberg, "Optogenetic and electrical microstimulation systematically bias visuospatial choice in primates," Curr. Biol. 24(1), 63-69 (2014).

64. J. Cavanaugh et al., "Optogenetic inactivation modifies monkey visuomotor behavior," Neuron 76(5), 901-907 (2012).

65. A. Watakabe et al., "Visualization of cortical projection neurons with retrograde TET-off lentiviral vector," PLoS One 7(10), e46157 (2012).

66. H. Adelsberger et al., "Local domains of motor cortical activity revealed by fiber-optic calcium recordings in behaving nonhuman primates," Proc. Natl. Acad. Sci. U. S. A. 111(1), 463-468 (2014).

67. G. Cui et al., "Concurrent activation of striatal direct and indirect pathways during action initiation," Nature 494(7436), 238-242 (2013).

68. Z. A. Peterlin et al., "Optical probing of neuronal circuits with calcium indicators," Proc. Natl. Acad. Sci. U. S. A. 97(7), 3619-3624 (2000).

69. L. Lo and D. J. Anderson, "A Cre-dependent, anterograde transsynaptic viral tracer for mapping output pathways of genetically marked neurons," Neuron 72(6), 938-950 (2011).

70. K. T. Beier et al., "Anterograde or retrograde transsynaptic labeling of CNS neurons with vesicular stomatitis virus vectors," Proc. Natl. Acad. Sci. U. S. A. 108(37), 15414-15419 (2011).

71. Y. Zhao et al., "An expanded palette of genetically encoded $\mathrm{Ca}(2)$ indicators," Science 333(6051), 1888-1891 (2011).

72. J. Y. Lin et al., "ReaChR: a red-shifted variant of channelrhodopsin enables deep transcranial optogenetic excitation," Nat. Neurosci. 16(10), 1499-1508 (2013)

73. N. C. Klapoetke et al., "Independent optical excitation of distinct neural populations," Nat. Methods 11(3), 338-346 (2014).

74. T. L. Lewis, Jr. et al., "Myosin-dependent targeting of transmembrane proteins to neuronal dendrites," Nat. Neurosci. 12(5), 568-576 (2009).

75. S. C. Rogan and B. L. Roth, "Remote control of neuronal signaling," Pharmacol. Rev. 63(2), 291-315 (2011).

76. C. J. Magnus et al., "Chemical and genetic engineering of selective ion channel-ligand interactions," Science 333(6047), 1292-1296 (2011).

Biographies of the authors are not available. 